\title{
Research on Pricing Decision of Closed-Loop Supply Chain Based on Carbon Tax
}

\author{
Zhang Kejing*, Ji Jing \\ Glorious Sun School of Business and Management, Donghua University, Shanghai, China
}

Email address:

zhangkj@dhu.edu.cn (Zhang Kejing),jijing1126@foxmail.com （Ji Jing）

To cite this article:

Zhang Kejing, Ji Jing. Research on Pricing Decision of Closed-Loop Supply Chain Based on Carbon Tax. International Journal of Economics, Finance and Management Sciences. Vol. 3, No. 5, 2015, pp. 623-629. doi: 10.11648/j.ijefm.20150305.36

\begin{abstract}
The closed-loop supply chain in this study is consist of a manufacturer, a retailer and a third-party collector of waste product. Under the decentralized decesion, the Stakelberg game is used to analyze the pricing strategy based on carbon tax. There sult show that carbon tax mechanism can effectively enhance collection volume and recycling, and all supply chain partners benefit from it.
\end{abstract}

Keywords: Carbon Tax, Closed-Loop Supply Chain, Stackelberg Game, Pricing Strategy

\section{碳税政策下的闭环供应链定价决策研究}

\section{张科静*，纪静}

旭日工商管理学院, 东华大学, 上海, 中国

\section{邮箱}

zhangkj@dhu. edu. cn (张科静)，jijing1126@foxmail.com（纪静）

摘要: 本文供应链由单一制造商、单一零售商和单一第三方回收商组成, 利用stackelberg博弯, 研究了分散决策下基 于碳税机制的闭环供应链定价策略。研究结果表明：基于碳税的机制能提升回收量, 可以有效促进闭环供应链回收再 制造。

关键词: 碳税, 闭环供应链, Stackelberg博弯, 定价决策

\section{1. 引言}

近年来，气候问题引起全球的关注，为解决温室气体 排放带来的环境问题，世界各国采用了多种政策手段来解 决。与此同时, 废旧产品的回收再制造活动越来越被认可 为一种创造盈利又环保的方式。电子产品更新换代快, 电 子产品回收再制造成为趋势。电子产品的回收再制造是一 种既兼顾经济效益又可降低碳排放、环保的生产方式。政 府利用怎样的管理方式或政策, 来影响闭环供应链更好的 回收再制造已经成为现实问题。
目前, 已经有不少学者研究了政府影响下的闭环供应 链定价决策问题。在碳税方面, Bettina B.F Wittneben 从减排量等几个方面论证了碳税在供应链中碳排放的影 响中更有效成本更低 [1]。Hua等在碳交易制度背景下, 从 库存的角度研究了碳交易价格和碳排放限额对企业订货 决策和碳排放的影响 [2]。安崇义等在碳排放交易机制下 研究了企业碳减排决策模型, 探讨了碳税的加入对排放权 交易市场的影响 [3]。Zhao等研究了排放配额分配和交易 机制下企业的技术选择问题, 分析配额对决策结果的影响 [4]。马秋卓等研究了以碳配额机制为基础的碳交易体系 下单个企业低碳产品最优定价和碳排放策略问题 [5]。 Ben jaafar在供应链系统中加入碳的因素, 建立碳排放限 
额模型、碳税模型、限额与交易模型等，给低碳供应链提 出很好的管理启示 [6]。常香云等对碳排放约束的企业制 造、再制造的最优生产决策进行了研究, 分析了碳税对企 业生产决策的影响 [7]。赵道致研究了碳税对供应商主导 的二级供应链减排博弯进行了研究, 求得了减排策略下企 业最优产量与单位产品减排量 [8]。岳晓娟在有无碳税两 种情况下探讨零售商最优单位产品碳排放和最优零售价 格的问题 [9]。李晓妮等研究了碳税政策对闭环供应链成 员定价决策影响, 得到碳税能够促进回收, 但对市场需求 有负面作用 [10]。高举红等基于补贴和碳税研究了闭环供 应链的定价策略, 得出碳税和补贴能够有效降低闭环供应 链碳排放、提高回收率 $[11]$ 。

在现有研究基础上, 本文以一个制造商、一个零售商 和一个第三方回收商组成的闭环供应链为研究框架, 拟考 察碳税对闭环供应链定价决策的影响, 并探讨政府的作用 是否会促进回收再制造问题。与现有文献的区别：对再造
品和新产品采用差别定价的方式, 在此基础上考虑碳税对 闭环供应链定价策略的影响。

\section{2. 问题描述}

本文讨论的闭环供应链是单周期的, 只考虑一个周期 的生产和销售情况, 忽略前后周期之间的相互影响, 前期 已有产品销售, 研究期可以回收再利用。如图1所示, 闭 环供应链单一制造商、单一零售商和单一第三方回收商组 成。制造商生产一种同质产品, 既可以完全使用原材料进 行生产, 也可以使用回收产品的部分零部件进行生产。制 造商通过零售商进行销售, 并通过第三方回收旧产品用于 再制造。为有效引导回收、降低碳排放量, 在分散决策下 本文考虑政府向制造商征收碳税, 其税率为 $f$ 。

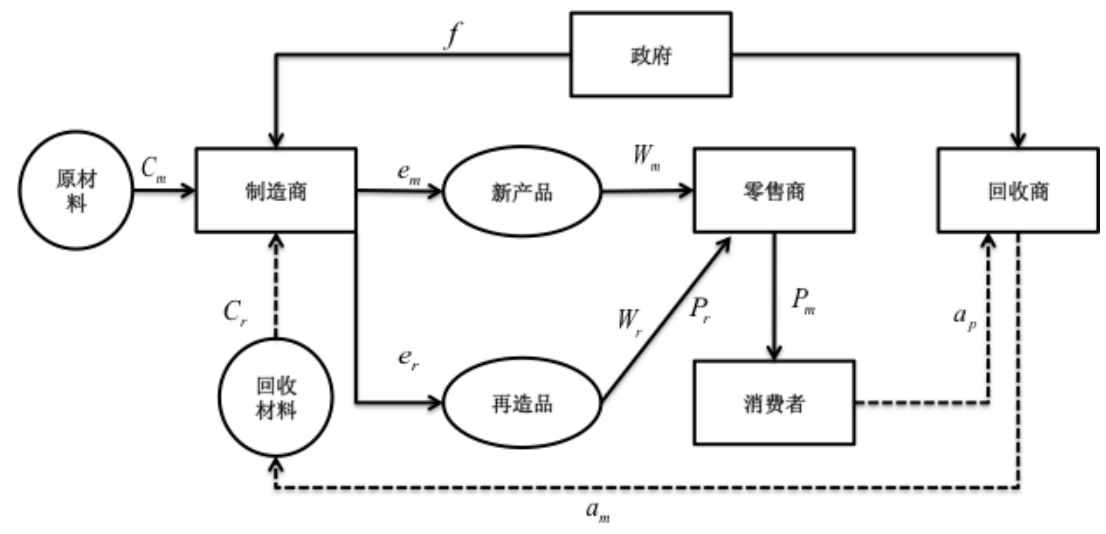

图1 闭环供应链回收再制造的决策结构。

\section{1. 参数设定}

$C_{m} 、 C_{r}$ 分别表示利用原材料和回收品（下文简称 新产品和再造品）生产的单位制造成本, $W_{m} 、 W_{r}$ 表示 新产品和再制品的单位批发价格, $P_{m} 、 P_{r}$ 表示新产品和 再制品的单位零售价格, $e_{m} 、 e_{r}$ 表示新产品和再制品的 单位碳排放量。 $a_{p}$ 表示回收商回收的单位价格, $a_{m}$ 表示 制造商从回收商处回收的回收转移成本。 $f$ 表示政府向制 造商征收的碳税税率。这里制造商的单位碳排放量 $e_{m} 、 e_{r}$ 都是固定的。

本文由于采用两种方式进行生产, 新产品和再制品的 市场需求各不相同。影响产品销售量的参数如下: $\alpha 、 \beta$ 、 $\gamma$ 、 $\tau$ 均为大于 0 的常数, $a$ 为潜在市场需求, $\tau$ 为新产 品所占总销量的比例, $\beta$ 为价格影响因子, $\gamma$ 为交叉价格 影响因子, 并且有 $\beta>\gamma$ 。

进一步地, 本文用 $\pi_{i}^{j}$ 表示为在决策模型 $i$ 中, 闭环 供应链中成员 $j$ 的利润。其中, $j=D, F$ 表示闭环供应 链决策模型、基于碳税的决策模型。 $i=M, R, 3 P$, 表示 制造商、零售商和第三方。

\section{2. 前提假设}

假设 1 : 单位回收品再制造成本 $C_{r}$ 低于单位新产品 的制造成本 $C_{m}$, 即 $C_{r}<C_{m}$ 。

假设 2 : 由于采用两种方式进行生产, 再制品的价格 会低于新产品价格, 即 $a_{m} \geq a_{p}$, 消费者在购买时会存在选 择偏好。本文假设新产品和再制品的市场需求分别是 $Q_{m}=\tau \alpha-\beta p_{m}+\gamma\left(p_{r}-p_{m}\right), \quad Q_{r}=(1-\tau) \alpha-\beta p_{r}+\gamma\left(p_{m}-p_{r}\right)$, 且有 $Q_{m} \geq 0, Q_{r} \geq 0$ 。

假设 3 : 第三方负责回收, 且回收量是关于回收价格 $a_{p}$ 的线性函数 $D_{P}=b+\lambda a_{p}$ 。其中, $b$ 表示回收价格为 0 时的 废旧品的市场供应量, 它的大小取决于消费者的环保意识, $b$ 越大表明消费者的环保意识越强， $\lambda$ 为价格影响因子。

假设 4 : 制造商生产阶段, 单位产品的碳排放量有 $e_{m}>e_{r}$ 。

假设5: 制造商及零售商是完全理性及风险中性的, 根据自身利润最大化原则来进行定价决策。

假设6: 碳税机制是政府为了确保闭环供应链降低碳 排放量水平而设计。 
假设7: 市场是完全开放的, 闭环供应链各成员都是在信 息对称的情况下做出的决策, 市场对产品的需求量相对稳定, 每周期的情况相类似, 故本文只研究单周期范围内的情况。

\section{3. 闭环供应链的回收再制造决策模型}

\section{1. 闭环供应链分散式决策模型}

不考虑碳税征收的情形, 闭环供应链成员制造商、零 售商和第三方的利润函数如公式（1）、（2）、（3）所 示:

$$
\begin{gathered}
\pi_{D}^{M}=\left(w_{m}-c_{m}\right) Q_{m}+\left(w_{r}-c_{r}\right) Q_{r}+\left(c_{m}-c_{r}-a_{m}\right) D_{p} \\
\pi_{D}^{R}=\left(p_{m}-w_{m}\right) Q_{m}+\left(p_{r}-w_{r}\right) Q_{r} \\
\pi_{D}^{P}=\left(a_{m}-a_{p}\right) D_{p}
\end{gathered}
$$

在这里，我们建立了制造商领导的非合作博弯模型， 制造商、零售商和第三方回收商进行两阶段动态博弯, 博 弯顺序如下: 正向渠道, 制造商先决定批发价格, $w_{m} 、 w_{r}$, 零售商根据制造商定的批发价来确定零售价格 $p_{m} 、 p_{r}$, 逆向渠道, 制造商首先确认从第三方回收商手中回收废旧 品, 并确认支付转移成本 $a_{m}$, 第三方回收商随之决定从消 费者手中回收废旧品, 回收价格为 $a_{p}$ 。在此模型中, 零售 商通过决策变量 $p_{m}$ 和 $p_{r}$ 的值来最大化自己的利润, 通过 对 (2) 式分别求 $p_{m}$ 和 $p_{r}$ 的一阶偏导数, 进一步在对其 求二阶导数求得海塞矩阵, $H=\left(\begin{array}{lr}-2(\beta+\gamma) & 2 \gamma \\ 2 \gamma & -2(\beta+\gamma)\end{array}\right)$ 为负定矩 阵, 所以 (2) 式为严格凹函数, 从而在驻点可以求得最优 解。联立方程, $\frac{\partial \pi_{D}^{R}}{\partial p_{m}}=0, \frac{\partial \pi_{D}^{R}}{\partial p_{r}}=0$ 求得在以制造商为领 导者的Stackelberg模型中, 对制造商给定的 $w_{m} 、 w_{r}$ 零 售商的最优零售价的反映函数, 见公式 (4) 和 (5):

$$
\begin{aligned}
& p_{m}=\frac{-w_{m} \beta^{2}-a \gamma-2 w_{m} \beta \gamma-a \beta \tau}{2 \beta(\beta+2 \gamma)} \\
& p_{r}=\frac{-w_{r} \beta^{2}-a \gamma-2 w_{r} \beta \gamma-a \beta}{2 \beta(\beta+2 \gamma)}
\end{aligned}
$$

对 (3) 式求一阶导数, 求得以制造商为领导者的 stackelberg模型中, 对制造商给定的 $a_{m}$ 第三方的最优回 收价格反映函数:

$$
a_{p}=\frac{1}{2} a_{m}-\frac{b}{2 \lambda}
$$

制造商预测零售商会根据 $p_{m}\left(w_{m}\right) 、 p_{r}\left(w_{r}\right) 、 a_{p}\left(a_{m}\right)$ 来 做出决策, 将其带入 (1) 式, 并分别求 $w_{m} 、 w_{r} 、 a_{m}$ 的 一阶偏导数及二阶导数, 得到海塞矩阵 $H=\left(\begin{array}{ccc}-(\beta+\gamma) & \gamma & 0 \\ \gamma & -(\beta+\gamma) & 0 \\ 0 & 0 & -\lambda\end{array}\right)$, 为负定矩阵, 所以

位严格凹函数, 因此在驻点有最优解。

在此基础上, 求得制造商为领导的模型中, 制造商的 最优定价见公式（7）和（8）, 回收商的回收转移成本见 公式（9）：

$$
\begin{aligned}
& w_{m}=\frac{c_{m} \beta^{2}+a \gamma+2 c_{m} \beta \gamma+a \beta \tau}{2 \beta(\beta+2 \gamma)} \\
& w_{r}=\frac{a \beta+c_{r} \beta^{2}+a \gamma+2 c_{r} \beta \gamma-a \beta \tau}{2 \beta(\beta+2 \gamma)} \\
& a_{m}=\frac{\lambda\left(c_{m}-c_{r}\right)-b}{2 \lambda}
\end{aligned}
$$

将公式 $(7) 、(8) 、(9)$, 带入 $p_{m}\left(w_{m}\right) 、 p_{r}\left(w_{r}\right)$ 、 $a_{p}\left(a_{m}\right)$ 得到零售商的最优零售价见公式 (10)、（11）, 回收商的最优回收价见公式（12）:

$$
\begin{aligned}
& p_{m}=\frac{c_{m} \beta(\beta+2 \gamma)+3 a(\gamma+\beta \tau)}{4 \beta(\beta+2 \gamma)} \\
& p_{r}=\frac{c_{r} \beta(\beta+2 \gamma)+3 a(\beta+\gamma-\beta \tau)}{4 \beta(\beta+2 \gamma)} \\
& a_{p}=\frac{\lambda\left(c_{m}-c_{r}\right)-3 b}{4 \lambda}
\end{aligned}
$$

将（7）-（12）式带入制造商、零售商及回收商的利 润函数、需求函数及回收函数中, 得到需求量公式 (13)、 (14), 回收量公式 (15), 以及最优利润函数见公式 (16)、 (17)、（18）:

$$
\begin{aligned}
& Q_{m}=\frac{1}{4}\left(c_{r} \gamma-c_{m}(\beta+\gamma)+a \tau\right) \\
& Q_{r}=\frac{1}{4}\left(a+c_{m} \gamma-c_{r}(\beta+\gamma)-a \tau\right) \\
& D_{p}=\frac{1}{4}\left(b+\left(c_{m}-c_{r}\right) \lambda\right)
\end{aligned}
$$

$$
\pi_{D}^{M}=\frac{b^{2}}{8 \lambda}-\frac{1}{4} c_{m} c_{r} \gamma+\frac{\beta+\gamma}{8}\left(c_{m}^{2}+c_{r}^{2}\right)+\frac{a}{4}\left(c_{r}(\tau-1)-c_{m} \tau\right)+\frac{a^{2}}{8 \beta(\beta+2 \gamma)}\left(\beta+\gamma-2 \beta \tau+2 \beta \tau^{2}\right)
$$




$$
\begin{aligned}
& \pi_{D}^{P}=-\frac{1}{8} c_{m} c_{r} \gamma+\frac{\beta+\gamma}{16}\left(c_{m}^{2}+c_{r}^{2}\right)+\frac{a}{8}\left(c_{r}(\tau-1)-c_{m} \tau\right)+\frac{a^{2}}{16 \beta(\beta+2 \gamma)}\left(\beta+\gamma-2 \beta \tau+2 \beta \tau^{2}\right) \\
& \pi_{D}^{3 P}=\frac{\left(b+\lambda\left(c_{m}-c_{r}\right)\right)^{2}}{16 \lambda}
\end{aligned}
$$

\section{2. 碳税机制下的闭环供应链分散式决策模型}

该情形下向制造商征收税率为 $f$ 的碳税, 引导闭环供应链达到一定的回收量水平。制造商、零售商和第三方的利 润如公式（19）、（20）、（21）：

$$
\begin{aligned}
& \pi_{F}^{M}=\left(w_{m}-c_{m}-f e_{m}\right) Q_{m}+\left(w_{r}-c_{r}-f e_{r}\right) Q_{r}+\left(c_{m}+f e_{m}-c_{r}-f e_{r}-a_{m}\right) D_{p} \\
& \pi_{F}^{R}=\left(p_{m}-w_{m}\right) Q_{m}+\left(p_{r}-w_{r}\right) Q_{r} \\
& \pi_{F}^{3 P}=\left(a_{m}-a_{p}\right) D_{p}
\end{aligned}
$$

供应链成员最优决策分析方式如上文 (3.1)，结果如表1所示。

表1 不同机制下的决策结果汇总。

\begin{tabular}{lll}
\hline 变量 & 不同机制下的决策结果 & \\
\cline { 2 - 3 } & 闭环供应链决策模型 & 碳税机制下的闭环供应链决策模型 \\
\hline$w_{m}$ & $\frac{1}{2} c_{m}+\frac{\alpha D}{2 C}$ & $\frac{1}{2} A+\frac{\alpha D}{2 C}$ \\
$w_{r}$ & $\frac{1}{2} c_{r}+\frac{\alpha E}{2 C}$ & $\frac{1}{2} B+\frac{\alpha E}{2 C}$ \\
$a_{m}$ & $\frac{\lambda\left(c_{m}-c_{r}\right)-b}{2 \lambda}$ & $\frac{\lambda(A-B)-b}{2 \lambda}$ \\
$p_{m}$ & $\frac{1}{4} c_{m}+\frac{3 \alpha D}{4 C}$ & $\frac{1}{4} A+\frac{3 \alpha D}{4 C}$ \\
$p_{r}$ & $\frac{1}{4} c_{r}+\frac{3 \alpha E}{4 C}$ & $\frac{1}{4} B+\frac{3 \alpha E}{4 C}$ \\
$a_{p}$ & $\frac{\lambda\left(c_{m}-c_{r}\right)-3 b}{4 \lambda}$ & $\frac{\lambda(A-B)-3 b}{4 \lambda}$ \\
$Q_{m}$ & $\frac{1}{4}\left(c_{r} \gamma-c_{m}(\beta+\gamma)+\alpha \tau\right)$ & $\frac{1}{4}(B \gamma-A(\beta+\gamma)+\alpha \tau)$ \\
$Q_{r}$ & $\frac{1}{4}\left(\alpha+c_{m} \gamma-c_{r}(\beta+\gamma)-\alpha \tau\right)$ & $\frac{1}{4}(\alpha+A \gamma-B(\beta+\gamma)-\alpha \tau)$ \\
$D_{p}$ & $\frac{1}{4}\left(b+\left(c_{m}-c_{r}\right) \lambda\right)$ & $\frac{1}{4}(b+(A-B) \lambda)$ \\
$\pi^{M}$ & $\frac{b^{2}}{8 \lambda}+\frac{\beta+\gamma+\lambda}{8}\left(c_{m}^{2}+c_{r}^{2}\right)+\frac{\alpha}{4}\left(c_{r}(\tau-1)-c_{m} \tau\right)$ & $\frac{b^{2}}{8 \lambda}+\frac{\beta+\gamma+\lambda}{8}\left(A^{2}+B^{2}\right)+\frac{\alpha}{4}(B(\tau-1)-A \tau)+\frac{\alpha^{2} F}{8 C}-\frac{\gamma+\lambda}{4} A B+\frac{b(A-B)}{4}$ \\
$\pi^{R}$ & $+\frac{\alpha^{2} F}{8 C}-\frac{\gamma+\lambda}{4} c_{m} c_{r}+\frac{b\left(c_{m}-c_{r}\right)}{4}$ & $-\frac{1}{8} A B \gamma+\frac{\beta+\gamma}{16}\left(A^{2}+B^{2}\right)+\frac{\alpha}{8}(B(\tau-1)-A \tau)+\frac{\alpha^{2} F}{16 C}$ \\
$\pi^{3 P}$ & $\frac{\left(b+\lambda\left(c_{m} c_{r}+\frac{\beta+\gamma}{16}\left(c_{m}^{2}+c_{r}^{2}\right)+\frac{\alpha}{8}\left(c_{r}(\tau-1)-c_{m} \tau\right)+\frac{\alpha^{2} F}{16 C}\right.\right.}{16 \lambda}$ & $\frac{(b+\lambda(A-B))^{2}}{16 \lambda}$ \\
\hline
\end{tabular}

其中: $A=c_{m}+f e_{m}, B=c_{r}+f e_{r}, C=\beta(\beta+2 \gamma), D=\beta \tau+\gamma, E=\beta+\gamma-\beta \tau, F=\beta+\gamma-2 \beta \tau+2 \beta \tau^{2}$ 


\section{3. 最优决策分析}

前文 $\left(2.2\right.$ 节) 中的假设需要满足 $Q_{m} \geq 0, Q_{r} \geq 0$,

$$
0 \leq f \leq \frac{\alpha \tau-\beta c_{m}+\gamma\left(c_{m}-c_{r}\right)}{\gamma\left(e_{m}-e_{r}\right)+e_{m} \beta}
$$

即, $c_{r} \gamma-c_{m}(\beta+\gamma)+\alpha \tau \geq 0 \alpha+c_{m} \gamma-c_{r}(\beta+\gamma)-\alpha \tau \geq 0$,

$(B \gamma-A(\beta+\gamma)+\alpha \tau) \geq 0, \quad \alpha+A \gamma-B(\beta+\gamma)-\alpha \tau \geq 0$

求得,

$$
\begin{aligned}
& 0<\beta \leq \frac{\alpha}{c_{m}+c_{r}} \\
& 0<\gamma \leq \frac{c_{m}(\tau-1)+c_{r} \tau}{c_{m}^{2}-c_{r}^{2}} \alpha \\
& 0 \leq f \leq \frac{\alpha-\beta\left(c_{m}+c_{r}\right)}{\left(e_{m}+e_{r}\right) \beta} \\
& \pi_{m}^{F}-\pi_{m}^{D}=\frac{\beta+\gamma+\lambda}{8}\left(2 f c_{m} e_{m}+2 f c_{r} e_{r}+f^{2} e_{m}^{2}+f^{2} e_{r}^{2}\right)+ \\
& \frac{a}{4}\left(f e_{r}(\tau-1)-f e_{m} \tau\right)-\frac{\gamma+\lambda}{4}\left(f c_{m} e_{r}+f c_{r} e_{m}+f^{2} e_{r} e_{m}\right)+\frac{b f\left(e_{m}-e_{r}\right)}{4} \\
& =\frac{\beta}{8}\left(2 f c_{m} e_{m}+2 f c_{r} e_{r}+f^{2} e_{m}^{2}+f^{2} e_{r}^{2}\right)+\frac{\gamma+\lambda}{8}\left(2 f e_{m}\left(c_{m}-c_{r}\right)+2 f e_{r}\left(c_{r}-c_{m}\right)+f^{2}\left(e_{m}^{2}+e_{r}^{2}-2 e_{r} e_{m}\right)\right) \\
& Q_{m}^{F}-Q_{m}^{D}=\frac{1}{4}\left(\gamma f\left(e_{r}-e_{m}-\beta e_{m}\right)\right) \leq 0
\end{aligned}
$$

在此前提下, 可以得到命题 1 -命题 3 :

命题1: $w_{m}^{F} \geq w_{m}^{D}, w_{r}^{F} \geq w_{r}^{D}, p_{m}^{F} \geq p_{m}^{D}, p_{r}^{F} \geq p_{r}^{D}$, $a_{m}^{F} \geq a_{m}^{D}, \quad a_{p}^{F} \geq a_{p}^{D}, \quad Q_{m}^{F} \leq Q_{m}^{D}, \quad D_{p}^{F} \geq D_{p}^{D}, \quad \pi_{m}^{F} \geq \pi_{m}^{D}$,

其中, $2 f e_{m}\left(c_{m}-c_{r}\right)+2 f e_{r}\left(c_{r}-c_{m}\right)$ 中, 因为 $c_{r}-c_{m}<0$, 所以,

$$
2 f e_{m}\left(c_{m}-c_{r}\right)+2 f e_{r}\left(c_{r}-c_{m}\right)>2 f e_{m}\left(c_{m}-c_{r}\right)+2 f e_{m}\left(c_{r}-c_{m}\right)=0
$$

$\pi_{m}^{F}-\pi_{m}^{D}$ 其余各式均大于零, 所以得到 $\pi_{m}^{F}-\pi_{m}^{D} \geq 0$

$$
\begin{aligned}
& \pi_{r}^{F}-\pi_{r}^{D}=-\frac{1}{8} \gamma\left(f c_{m} e_{r}+f c_{r} e_{m}+f^{2} e_{r} e_{m}\right)+\frac{a}{4}\left(f e_{r}(\tau-1)-f e_{m} \tau\right) \\
& +\frac{\gamma+\beta}{16}\left(2 f c_{m} e_{m}+2 f c_{r} e_{r}+f^{2} e_{m}^{2}+f^{2} e_{r}^{2}\right) \\
& =\frac{\beta}{16}\left(2 f c_{m} e_{m}+2 f c_{r} e_{r}+f^{2} e_{m}^{2}+f^{2} e_{r}^{2}\right)+\frac{\gamma}{16}\left(2 f e_{m}\left(c_{m}-c_{r}\right)+2 f e_{r}\left(c_{r}-c_{m}\right)+f^{2}\left(e_{m}^{2}+e_{r}^{2}-2 e_{r} e_{m}\right)\right) \\
& +\frac{a}{4}\left(f e_{r}(\tau-1)-f e_{m} \tau\right)
\end{aligned}
$$

上式中前两个多项式都为正, 最后一个多项式为负。 因此，零售商在基于碳税机制下的利润与政府影响机制的 利润相比, 受 $\alpha$ 市场潜在需求, 新产品所占市场份额及价 格影响因子及交叉价格影响因子影响。

命题2: 闭环供应链各个成员的决策变量都是关于碳 税的增函数, 政府碳税的征收, 新产品与再造品的单位批 发价和零售价都随着碳税的增加而增加, 回收价格及制造 商回收的转移成本也为增函数。

对批发价 $w_{m} 、 w_{r}$, 零售价 $p_{m} 、 p_{r}$, 回收价 $a_{m}$ 、 $a_{p}$, 求关于碳税 $f$ 的偏导数, 求得:

$$
\begin{aligned}
\frac{\partial w_{m}}{\partial f}=\frac{1}{2} e_{m}>0, \frac{\partial w_{r}}{\partial f} & =\frac{1}{2} e_{r}>0, \frac{\partial p_{m}}{\partial f}=\frac{1}{4} e_{m}>0, \\
\frac{\partial p_{r}}{\partial f} & =\frac{1}{4} e_{r}>0, \\
\frac{\partial a_{m}}{\partial f}=\frac{1}{2}\left(e_{m}-e_{r}\right) & >0, \frac{\partial a_{r}}{\partial f}=\frac{1}{4}\left(e_{m}-e_{r}\right)>0
\end{aligned}
$$

命题1和命题2说明政府通过征收碳税, 增加了生产成 本, 制造商通过提高批发价来保证其收益, 零售商随之提 高零售价格。碳税的影响, 使得制造商更愿意利用废旧品 进行生产, 从而影响回收商的回收量增加, 回收价格也随 之增加, 新产品的产量降低, 废旧品的产量增加。 
命题3: 产品的销售总量与新产品的销售量是关于碳 税的减函数, 再造品的销量除与碳税有关外, 还与价格影 响因子 $\beta$ 以及新产品和再造品的单位碳排放 $e_{m} 、 e_{r}$ 的差 值有关。

证明：对以下各式求关于碳税的偏导数, 求得:

$$
\begin{gathered}
\frac{\partial\left(Q_{m}+Q_{r}\right)}{\partial f}=-\frac{\beta}{4}\left(e_{m}+e_{r}\right)<0, \quad \frac{\partial Q_{m}}{\partial f}=\frac{\gamma e_{r}-(\gamma+\beta) e_{m}}{4}<0, \\
\frac{\partial Q_{r}}{\partial f}=\frac{\gamma e_{m}-(\gamma+\beta) e_{r}}{4}
\end{gathered}
$$

当上式大于零时, 再造品的销售量增加, 当上式小于 零, 再造品的销售量减少。

$$
\frac{\partial D_{p}}{\partial f}=\frac{\gamma\left(e_{m}-e_{r}\right)}{4}>0
$$

命题 3 表明, 碳税的征收导致生产成本增加, 批发价 及零售价都随之提高, 市场的总需求因而下降, 但消费者 的消费行为受到零售价格的影响, 原先有计划购买新产品 的顾客消费行为发生了改变, 再造品的市场需求增加, 这 部分是交叉价格影响因子的作用。

与此同时, 制造商从成本和市场两个角度, 更加关注 再造品的生产。废旧商品的回收量会随之增加, 制造商会 更注重回收和环保, 制定合理的生产及回收计划使由碳税 增加导致的利润损失降低到最小。从环境保护角度看待政 府碳税征收问题, 碳税的征收必然导致回收价格提高, 回 收量增加, 生产方面的碳排放量减少, 对于碳排放减少方 面有积极的影响。从市场方面来分析, 碳税征收, 市场的 销售价增加, 市场需求减少, 导致市场不活跃。

总之, 政府在征收碳税方面, 应该确定一个合适的范 围, 综合考虑环境和经济两个方面的影响。

\section{4. 算例分析}

设某电子产品闭环供应链的相关参数为 $c_{m}=10 、 c_{r}=5$ 、 $e_{m}=0.5 、 e_{r}=0.2 、 \alpha=100 、 \tau=0.7 、 \beta=3 、 \gamma=0.5 、 b=10 、 \lambda=8$ 、 $D_{0}=2$ 。

表2 两种情形下的决策结果。

\begin{tabular}{llllllll}
\hline 碳 & \multicolumn{7}{l}{ 不同机制下的决策结果 } \\
\cline { 2 - 8 } 税 & $D_{p}$ & $a_{m}$ & $a_{p}$ & $\pi^{M}$ & $\pi^{R}$ & $\pi^{3 P}$ & $\pi$ \\
\hline 0 & 12.5 & 1.88 & 0.31 & 83.33 & 34.65 & 19.53 & 137.51 \\
3 & 14.3 & 2.36 & 0.54 & 94.04 & 31.44 & 25.56 & 151.04 \\
6 & 16.1 & 2.78 & 0.76 & 108.35 & 38.49 & 32.40 & 179.24 \\
9 & 17.9 & 3.23 & 0.99 & 126.26 & 55.8 & 40.05 & 222.11 \\
12 & 19.7 & 3.68 & 1.21 & 147.77 & 83.37 & 48.51 & 279.65 \\
15 & 21.5 & 4.16 & 1.44 & 172.88 & 121.2 & 57.78 & 351.86 \\
18 & 23.3 & 4.58 & 1.66 & 201.59 & 169.29 & 67.86 & 438.74 \\
21 & 25.1 & 5.03 & 1.89 & 233.9 & 227.64 & 78.75 & 540.29 \\
24 & 26.9 & 5.46 & 2.11 & 269.81 & 296.25 & 90.45 & 656.51 \\
\hline
\end{tabular}

首先比较两种情形的决策结果, 然后通过算例讨论不 同机制下的制造商、零售商和第三方的利润随参数改变的
变化情况。由式 (24) 和 (25) 知, 碳税需要满足 $f \leq 25.76$, 所以设置本文碳税范围为 $[0,24]$. 所得计算结果, 如表 2 。

从表 2 可以看出, 基于碳税的机制, 批发价和零售价 提高, 回收价格和回收转移成本增大, 回收量增多, 产品 总碳排放量下降, 零售商利润降低, 制造商利润增大, 闭 环供应链总利润提高。

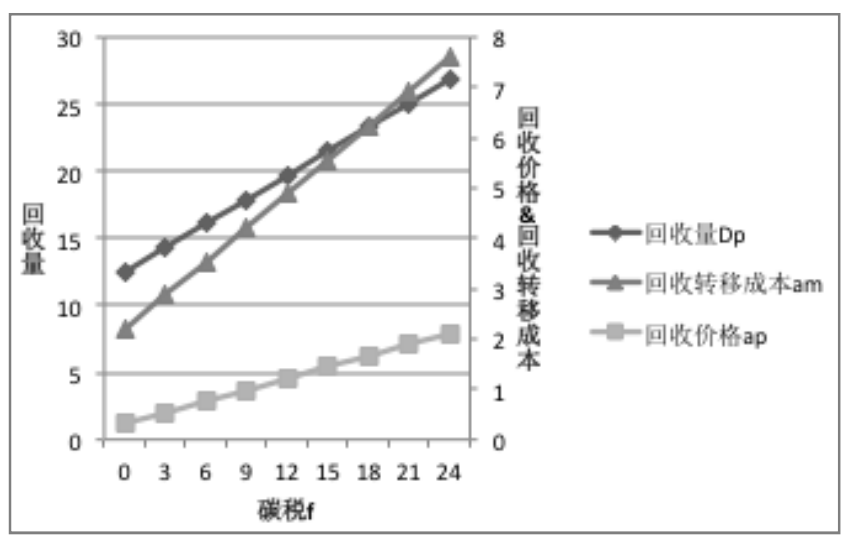

图1 碳税对回收量、回收价格及回收转移成本的影响。

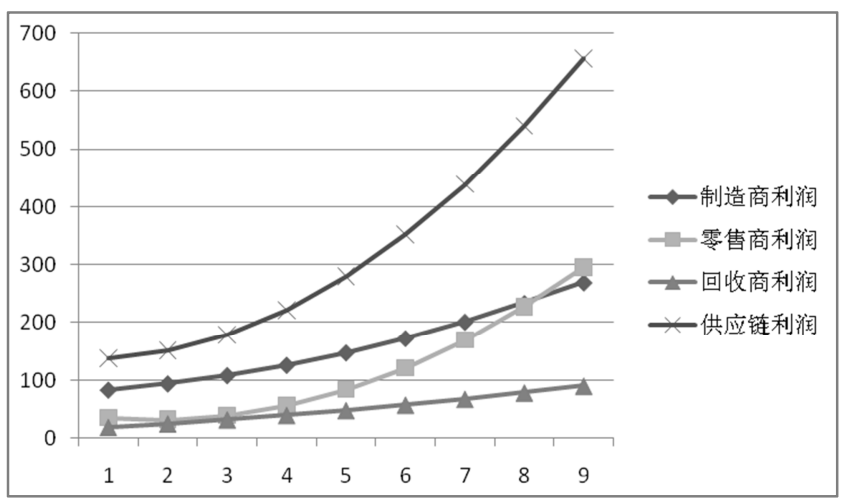

图2 碳税对供应链利润的影响。

从以上图表可以得到, 基于碳税的机制能够有效引导 回收, 回收再制造节约成本, 提高供应链的利润。

\section{5. 结束语}

本文基于碳税机制, 应用Stackelberg博弯, 研究了 由一个制造商、一个零售商及一个第三方回收商组成的两 级闭环供应链定价模型。以分散决策为基准, 建立了闭环 供应链在碳税机制下的定价策略。采用具体的数值算例分 析了碳税力度对回收量、回收价格、回收转移成本的影响, 验证了模型的有效性。

碳税机制, 提高了生产成本, 从而提高批发价和零售 价, 促进回收再制造, 回收量增多, 促进回收, 增加供应 链成员的利润, 对供应链的影响是积极的。

\section{致谢}

本研究受教育部人文社会科学研究规划基金项目的 资助（项目编号12YJA630185）。 


\section{参考文献}

[1] Bettina B.F. Witneben. Exxonisright: Letusre-examine our choice for a cap-and trade system over a carbon tax [J]. Energy Policy, 2009, 37 (6) : 2462-2464.

[2] Hua G W, Cheng T C E, Wang S Y.Managing Carbon Footprints in Inventory Management [J]. International Journal of Production Economics, 2011, 132:178-185.

[3] 安崇义, 唐跃军. 排放权交易机制下企业碳减排的决策模型 研究 $[J]$. 经济研究, 2012 (8) : 45-58.

[4] Zhao J, Hobbs B F, Pang J S. Long-Run Equilibrium Modeling of Emissions Allowance Allocation Systems in Electric Power Markets [J]. Operations Research, 2010, 58 (3) : 529-548.

[5] 马秋, 宋海清, 陈功玉. 碳配额交易体系下企业低碳产品定 价及最优碳排放策略 [J]. 管理工程学报, 2014, 2 (28) : 127-136。
[6] Saif Benjaafar, Yanzhi Li, Mark Daskin. Carbon Footprint and the management of Supply Chains: Insights from Simple Models [J]. IEEE Transactions on Automation Science and Engineering, 2012 (1):99.

［7］常香云, 朱慧赞. 碳排放约束下企业制造/再制造生产决策 研究 [J].科技进步与对策，2012，29（11）:75-78。

[8] 赵道致, 勾杰. 考虑碳税征收的供应链纵向减排博弯研究 [J]标准科学, 2013，11：53-57。

[9] 岳晓娟. 考虑两次碳排放的零售商定价策略研究 [D]西南交 通大学, 2013 。

[10] 李晓妮, 韩瑞珠. 碳税政策对闭环供应链成员定价决策影响 研究 $[J]$.物流工程与管理，2014，12：75-79。

[11] 高举红, 王海燕, 孟燕莎. 基于补贴与碳税的闭环供应链定 价策略 $[J]$ 工业工程, 2014，17（3）:61-68。 